\title{
Kearns Sayre Syndrome: A Mitochondrial Disorder
}

\author{
Anali Cisneros ${ }^{1}$ and Jeffrey O. Henderson ${ }^{1,2}$ \\ ${ }^{1}$ Department of Science and Mathematics, Judson University, Elgin, IL 60123, USA \\ ${ }^{2}$ Corresponding author: jeffrey.henderson@judsonu.edu \\ DOI: https://doi.org/10.47611/jsr.v10i1.1099
}

\section{ABSTRACT}

Kearns-Sayre syndrome (KSS) is a pleiotropic disorder caused by non-specific spontaneous deletion of a large amount of genetic material from mitochondrial DNA (mtDNA). Aside from patients having mtDNA defects there are also autosomal mutations in nuclear DNA, indicating KSS is not caused by a single gene mutation, but is most likely the result of mutations in genes forming a common biochemical pathway. KSS is characterized by a wide array of symptoms including: ophthalmoplegia, pigmentary retinopathy, ataxia, cardiac conduction defects that later develop into cardiac complications, and brain abnormalities. This review considers the association of deletions in mtDNA with a decrease in mitochondrial function and the pathogenetic role of the dysfunctional mitochondria by analyzing different variants of the mitochondrial genome. Despite there being no curative treatment for these patients, some possible disease modifying therapies have been proposed such as folinic acid supplementation and intravenous arginine therapy.

\section{Introduction}

Human mitochondrial DNA (mtDNA) is a double-stranded 16.5 kilobase circular molecule with no introns that encodes 37 genes including 13 protein coding genes, 22 transfer RNA genes, and 2 ribosomal RNAs genes encoding the 16S and 12S mitochondrial ribosomal RNAs (Zhang et al., 2017; Patananan et al., 2017). The 13 peptides that mtDNA encodes are components of respiratory-chain complexes which explains why the disorder has such high correlation with cardiac manifestations (Moraes et al., 1989). Due to the high requirement of oxygen levels needed by physiological processes in the body, the deficient energy production caused by defective mitochondria causes the cardiac muscle to compensate by increasing the number of mitochondria (mitochondria proliferation) which leads to cellular swelling and myocardial hypertrophy (Guenthard et al., 1995). About 10-15\% of mitochondrial disorders (MD) are caused by mutations in mtDNA while $25 \%$ of children with MD have nuclear DNA mutations (Chi, 2015). MD can be determined by biochemical abnormalities of the mitochondria. Muscle biopsies can also reveal the presence of ragged red fibers on trichome stain as well as the excessive accumulation of mitochondria (Moraes et al., 1989; Khambatta et al., 2014). Interestingly, more than 200 nuclear encoded genes have been linked with children affected by MD.

Among patients with KSS, all patients present with a 45-75\% deletion of the mtDNA genome and the common deletion site for patients with KSS is a $4.9 \mathrm{~kb}$ fragment. This fragment is found within nucleotides $8470-8482$ and 13,447-13,459 (Moraes et al., 1989; A. Saldaña-Martinez et al., 2019). These large deletions of mtDNA have been correlated with cardiac conduction defects. The severity of KSS is positively correlated with the size and heteroplasmy of the large-scale deletion (A. Saldaña- Martinez et al., 2019). Heteroplasmy results when cells contain a combination of mutant and wild-type mtDNAs. An investigation done by Moraes and colleagues (1988) found that two KSS patients had both deleted and normal mtDNAs present together in affected tissues. Thus, the results imply that at least some deleted mtDNAs are segregated in organelles that are devoid of wild-type mtDNAs (Nakase et al., 1990). Furthermore, KSS patients have ratios of mutated to normal mitochondrial DNAs highest in skeletal muscle 
followed by heart, kidney, and liver tissue. Therefore, variance in organ involvement in KSS may be due to the uneven proportion of wild-type and mutant DNAs in diverse tissues (Anan et al., 1995).

Diagnosis for KSS can be done through clinical impression by observing common and easily detected symptoms of KSS in the patient such as bilateral ptosis, a drooping of the eyelids. Considering the rapidly expanding phenotypic spectrum of clinical features and phenotypic expression among affected patients, the risk for under diagnosis and delay for appropriate referrals is very high when done through clinical impression. Additionally, because a variety of genetic mutations deleteriously affect mitochondrial function it can be very daunting to make a comprehensive diagnosis (Gorman et al., 2015). The clinical presentations of mitochondrial disorders have common overlaps between Mitochondrial Encephalomyopathy, Lactic Acidosis and Stroke-like episodes syndrome (MELAS) and KSS (Yu, et al., 2016). Mitochondrial encephalomyopathy is defined by disease, damage, or malfunction of the brain indicating that KSS patients have a severely affected central nervous system (CNS) (Quijada-Fraile et al., 2014). Thus, laboratory testing needs to be performed in conjunction with a clinical examination that analyzes features like elevated cerebrospinal fluid protein and low folate levels that are characteristic of KSS patients and can confirm the diagnosis. Patients that do present with ophthalmoplegia and ptosis but do not have an established diagnosis of KSS need to be routinely screened for Atrioventricular Block (AV) as it typically manifests after the ophthalmologic abnormalities (Young et al., 2005). This cardiac conduction disturbance- AV block occurs when the electrical signal that controls the heartbeat is completely occluded and is associated with a patient mortality of $20 \%$. Another common irregularity involved in the cardiac pathology of KSS observed on electrocardiograms include His-ventricular (H-V) interval prolongation which results from dilated cardiomyopathy (Young et al., 2005). H-V interval is defined as abnormal when it exceeds 55 milliseconds and has been correlated with the mtDNA deletions within the cardiomyocytes provoking dilated cardiomyopathy in KSS (Anan et al., 1995).

The prevalence rate for adults with a mitochondrial disease caused by either the nuclear or mitochondrial genome is 12.5 in 100,000 and for KSS specifically it is estimated to be 1 to 3 per 100,000 individuals (Gorman et al., 2015). The mean age of KSS symptom presentation is 17 years; however, the mean age at diagnosis is 26 years due to difficulty in assigning a diagnosis to this pleiotropic disease (Khambatta et al., 2014). Patients typically under the age of 20 years present with an onset of cerebellar ataxia, cardiac conduction defects, pigmentary retinopathy and chronic progressive ophthalmoplegia (Moraes et al., 1989). Out of the 23 patients analyzed in a study done by Broomfield (2015), 47\% of patients had the most common initial presentation of KSS, isolated ptosis, and $32 \%$ had transfusion-dependent anemia soon after birth (Broomfield et al., 2015). The ten presenting with significant anemia fulfilled the criteria for a confirmed diagnosis of KSS. When diagnosing a strictly pediatric aged patient, a muscle biopsy is not necessary as support for the diagnosis, since detection of single large-scale mitochondrial DNA (mtDNA) deletions (SLSMDs) can be found by doing molecular diagnosis via long-range polymerase chain reaction or Southern blotting of DNA isolated from blood leukocytes or urinary tract epithelial cells (Broomfield et al., 2015). N. Yu and colleagues (2016) compared the levels of mtDNA mutations in blood cells versus urinary epithelial cells in a patient. In order to increase the amounts of urinary epithelial cells the $350 \mathrm{~mL}$ sample was collected after a 10 min exercise in the morning. Their results demonstrated a heteroplasmic ratio of mutant to normal mtDNA in leukocytes versus urinary epithelial cells at $15 \%$ versus $78 \%$, respectively. These data demonstrate a comparatively high frequency of renal disease in patients with mtDNA deletions allowing for the use of urinary markers to detect renal involvement and early intervention for patients with KSS (Broomfield et al., 2015). Therefore, the less invasive blood specimens or urinary markers can be used as an alternative to muscle biopsies for molecular diagnosis since patients usually present with mutant mtDNA in all tissues analyzed (Wong, 2001).

\section{Inheritance}

Mitochondria are contributed exclusively by the ovum's cytoplasm during the formation of the zygote; therefore, the mitochondrial genome is maternally transmitted by a non-Mendelian mechanism (Wallace, 1999). Consequently, an affected mother would pass the disease to all her children, but only her daughters would transmit the mtDNA mutation 
to subsequent generations; a hallmark of maternal plastid inheritance (Moraes et al., 1989). However, recent evidence has revealed that mitochondrial diseases can have a wide variety of inheritance patterns: simple Mendelian, maternal, or a combination of the two explaining their overlapping spectrum of disease (Wallace, 1999). Primary mtDNA mutations may display maternal inheritance, or Mendelian traits caused by numerous somatic mtDNA alterations. Since all mitochondria come exclusively from the oocyte, normal cells are homoplasmic, having only a single mtDNA genotype; in contrast, heteroplasmic cells present with a mixture of mitochondrial genomes. Additionally, segregation of mitochondria during mitosis influences cellular pathophysiology due to stochastic redistribution of wild-type and mutated genomes during mitochondrial and cell divisions (Chi, 2015).

Chinnery and colleagues (2004) hypothesize that the orignal pathogenetic deletion most likely arises in the oocyte before fertilization and is subsequently transmitted to the zygote. This hypothesis (Hypothesis 1; Figure 1) was proposed after observing a pair of identical twins that had discordancy for ophthalmoplegia with the affected brother having homoplasmy of a single deletion mutation in his mtDNA of skeletal muscle origin, with the other brother having heteroplasmy of the same deletion and being unaffected. This result is expected if the deletion arose in the oocyte as opposed to the same deletion arising independently in each zygote.

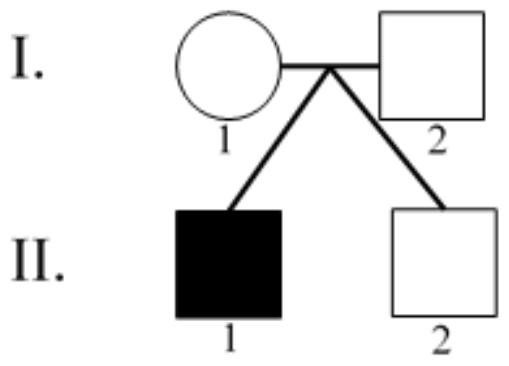

Figure 1. Hypothesis 1 Pedigree. The affected brother (II-1; filled square) having the single deletion of mtDNA present in skeletal muscle and the unaffected brother (II-2, open square). The mother (I-1) and father (I-2) are unaffected (open circle and open square, respectively).

However, if the source of deletions responsible for the disease is transmitted by healthy oocytes why is there no association between maternal age and the risk of having a child with a mtDNA deletion having KSS? There appears to be no correlation noted between maternal age and the risk of unaffected mothers having children with KSS indicating that the incidence of disruptive mtDNA deletions doesn't increase with maternal age. Another possible hypothesis (Hypothesis 2; Figure 2) is that the pathogenic mtDNA deletion is contained in oocytes from the maternal grandmother and exhibits matrilineal transmission. The female germ line appears to contribute significant changes in the level of mutant mtDNA, and thus mutant mitochondria, when females transmit heteroplasmic mtDNA mutations. Evidence for the contrarian view that mtDNA deletions arise spontaneously in an oocyte or the zygote is supported by the absence of affected mothers or siblings of KSS patients with detectable mtDNA deletions as determined by examining muscle-biopsy specimens (Moraes et al., 1989). 


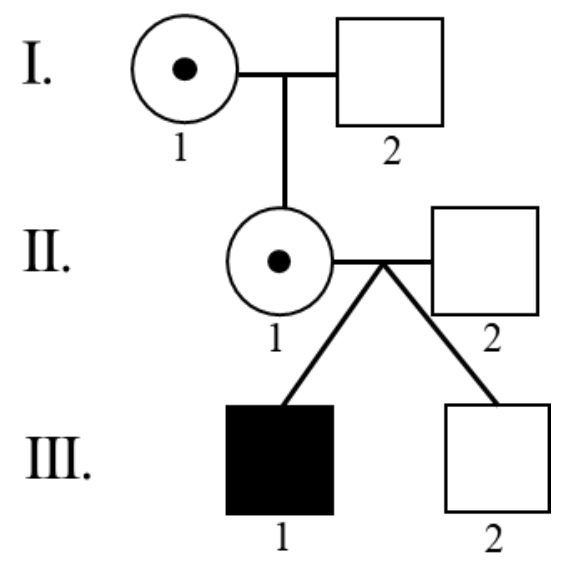

Figure 2. Hypothesis 2 Pedigree. This pedigree includes the maternal grandmother having oocytes carrying the single deletion of mtDNA revealing matrilineal transmission to the affected twin brother. As in Figure 1, the affected brother (III-1; filled square) having the single deletion of mtDNA present in skeletal muscle and the unaffected brother (III2, open square). The grandmother (I-1) and mother (II-1) are both carriers and unaffected (open circle with dot). The grandfather (I-2) and father (II-2) are unaffected (open square).

Since this disease is maternally inherited the affected mother transmits to her children by which the daughters then pass to subsequent generations. Thus, males and females are affected in equal numbers assuming complete penetrance (e.g. homoplasmy or biased heteroplasmy). Currently there is no racial predilection for the disorder. Additionally, it is not known what genetic mechanisms predispose an unaffected woman to have an affected child; however, women who exhibit heteroplasmy are highly unlikely to have more than one affected child as opposed to affected (homoplasmic) women who, on average, have a 4\% chance of delivering a clinically affected child (Chinnery et al., 2004). mtDNA function and structural integrity can be compromised due to nuclear gene defects leading to an authentic risk of disease recurrence for families with an affected individual. Therefore, a proportion of the unaffected children of affected mothers might develop symptoms in the future producing a higher incidence risk than children born to unaffected mothers (Chinnery et al., 2004).

In order to better understand KSS genetic ancestry through the maternal line, phylogenetic analysis was performed with 51 Amerindian and 84 Asian patients (A. Saldaña- Martinez et al., 2019). The authors performed a comprehensive multiple sequence alignment of complete mtDNA from haplogroup B for KSS patient 2 and haplogroup C for patients 1 and 3. The phylogenetic tree for patient 1 displayed haplogroup C1b14, concordant with the patient's mother, that traced back to an ancient Mayan individual indicating haplogroup $\mathrm{C}$ was present in Mexican pre-Hispanic populations. However, the data also reveals that haplogroup $\mathrm{C}$ is only present in very low frequencies in the Zapotec (present-day Mexican population), indicating it has not expanded into current populations. Further analysis done by A. Saldaña- Martinez and colleagues (2019) determined other haplogroup and mitochondrial pathogenic variants that influence the function of the mitochondria (Figure 3). The identification of the haplogroups was determined to define whether there exists any relevance to KSS since it has been previously shown in past studies that there are haplogroups associated with other disorders. For example, in the whole mitochondrial genome sequencing performed by SaldañaMartinez and colleagues (2019), 28 synonym variants were found to be statistically correlated with diseases such as cancer, Parkinson's disease, metabolic syndrome and optic neuropathy. Specifically, the variant m.8701G/10398G has been identified in two patients and has been shown to play a role in the pathophysiology of complex diseases by impacting mitochondrial matrix pH and intracellular calcium dynamics (A. Saldaña-Martinez et al., 2019). 


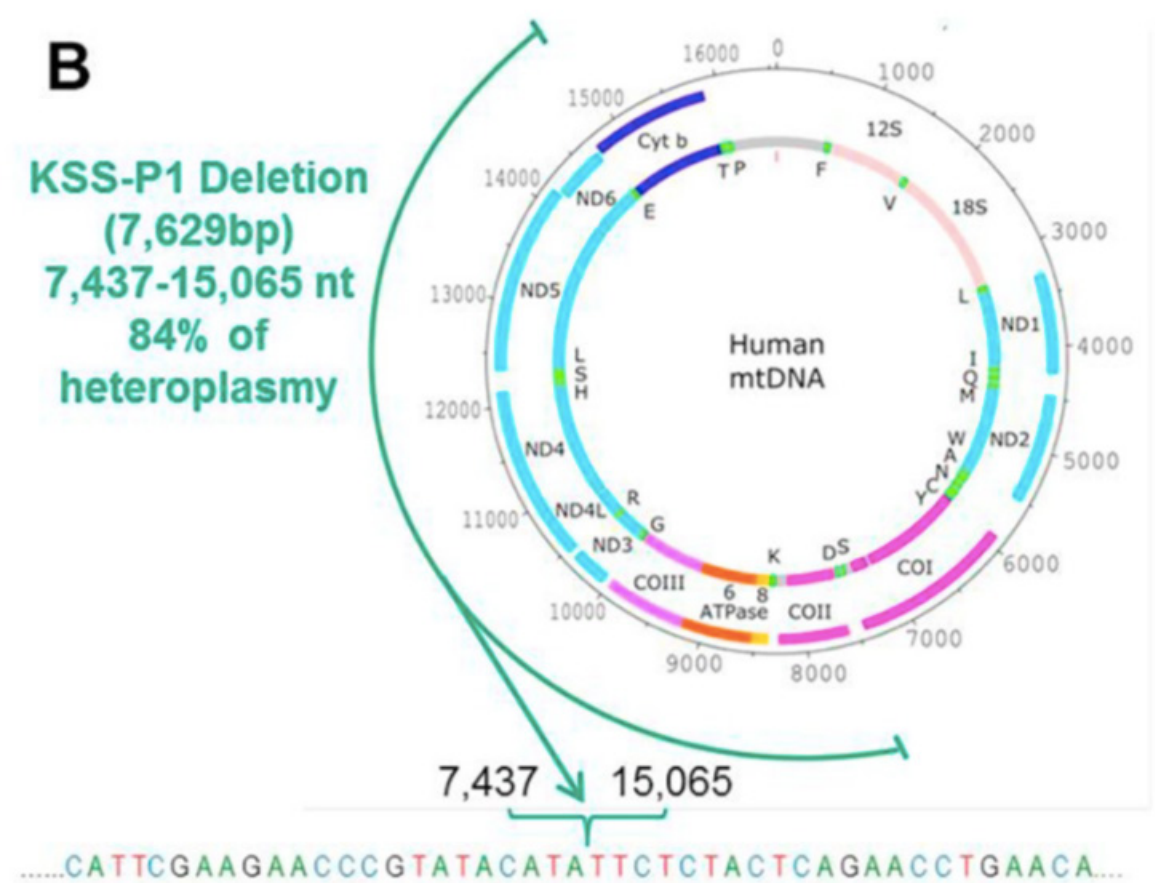

Figure 3. Characterization of the mtDNA deletion of KSS patient 1. This deletion is 7,629 base pairs and is highlighted by a green arrow. This figure is from A. Saldaña- Martinez et al., 2019.

Furthermore, depending on the variants present in the mitochondrial genome, the response to treatment varies providing insight into what medical treatments particular KSS patients will more likely respond to. By revealing mitochondrial variations we can better understand their influence on the total phenotype of organisms from general physiology to longevity. The multisystem nature of KSS affects multiple organ systems, showing that when there is sufficient proliferation of mutant mtDNA during mtDNA replication there is a higher rate of mutation versus when it occurs by random segregation. Knowing the mtDNA haplogroup for a KSS patient is non-trivial as it has been demonstrated that some mtDNA haplogroups can increase transcription and replication rates resulting in proliferation of mtDNA molecules. Identification of specific MD associated mtDNA haplogroups will contribute to treatment using human oocyte mitochondrial replacement therapy by identifying compatible donors through "mtDNA fingerprinting" (Young et al., 2005).

\section{Underlying Genetics}

Evidence suggests that KSS is a pleiotropic disorder since patients with identical deletions can have different clinical phenotypes. The sporadic occurrence of KSS indicates that the site and size of deletion can differ among patients, but all affected genomes still preserve the promoters for the transcription of heavy and light strands, the 12S and 16S ribosomal RNA genes, and the origin of heavy-strand replication (Moraes et al., 1989). Thus, the mutant mtDNA has to be capable for replication in order to explain the high proportion of genomes with deletions. The transcription of mitochondrial DNA is polycistronic. Therefore, the genes encoded on the heavy and light strands are transcribed as two large RNAs. These polycistronic RNAs are then processed into individual transfer and messenger RNAs (Moraes et al., 1989). Consequently, a deletion in mtDNA will most likely remove several cistrons (genes) causing disruptions in transcription and translation even if the genes are not directly adjacent to the deletion.

In an analysis of mtDNA from two KSS patients harboring different deletions, Southern blotting analysis of muscle DNA from case 1 showed a wild type $16.6 \mathrm{~kb}$ fragment as well as a $11.6 \mathrm{~kb}$ fragment corresponding to a 5.0 
$\mathrm{kb}$ deletion. As for case 2 aside from the wild type mtDNA $16.6 \mathrm{~kb}$ fragment, it had a $9.1 \mathrm{~kb}$ fragment corresponding to a $70 \%$ deletion of total mtDNA. In order to map out the deletions, direct sequencing of PCR products was performed. Case 1 had a 4,977 bp long deletion whereas case 2 had a 7,521 bp long deletion (Nakase et al., 1990). Since both of the deletions had perfect direct repeats of 8-13bp occurring in the same region, this site is considered a 'hot spot' prone for extensive deletions of mtDNA (Moraes et al., 1989). The results of this study reveal that the deleted mtDNAs are transcriptionally active but the mitochondrial ribosomes are unable to translate the mtRNAs, implying that only translation products derived from the wild type mtDNAs are active. These data indicate that perturbations in translation are critical to the pathogenesis of KSS (Nakase et al., 1990).

Furthermore, an investigation done by Anan and colleagues (1995) determined that there is a correlation found among specific mitochondrial mutations and cardiac abnormalities. Southern blot analysis was performed to detect mtDNA deletions by digestion with restriction enzymes PvuII, XbaI, HindIII, BamHI, and EcoRI to linearize the DNA molecules. After performing gel electrophoresis, the DNA fragments were blotted and probed with labeled whole mtDNA isolated from human liver; the deleted mtDNAs were mapped by examination for the absence of known restriction sites in the mtDNA (Anan et al., 1995). All three patients with KSS had a large deletion corresponding to positions 8433 bp to 13483 bp known as the 'hot spot' and had a proportion of deleted mtDNA ranging from 34.2\% and $60.3 \%$. Additionally, they showed disturbances in cardiac conduction and had heteroplasmy of mitochondrial DNA. Fifty-Seven percent of KSS patients present with AV cardiac conduction disturbance which is strongly correlated with the 'hot spot' deletion; the 'hot spot' deletion is found in $15-40 \%$ of heart muscle cell genomes (Anan $e t$ al., 1995).

\section{External Ophthalmoplegia}

All patients with KSS have eye involvement due to the high energy requirements of eye muscle cells; mitochondria make up $60 \%$, by volume, of extraocular muscle cells making these cells especially sensitive to mitochondrial perturbations (Khambatta et al., 2014). Thus, it is no surprise that the most common abnormality present in any patients with KSS is ophthalmoplegia, accompanied with ptosis and pigmentary retinopathy. Chronic progressive external ophthalmoplegia (CPEO) is characterized by weakness in the extraocular muscles that hold the eye in position and control its movement and typically starts out as ptosis (Sang-Jun Lee et al., 2018). This can be diagnosed by an ophthalmological evaluation that involves retinal examinations, such as an ophthalmoscopy (Lee et al., 2018). The most typical features of KSS onset before the age of 20 are CPEO, pigmentary retinopathy, and complete heart block in which the heart beats too slowly (Anan et al., 1995). In a study done by Lee and colleagues, of 16 male patients, 6 patients had ophthalmoplegia (38\%) and six had ptosis (38\%) as the most prevalent symptoms.

\section{Cardiovascular Complications}

Mutations (point, deletions, inversions etc.) of the mitochondrial and nuclear genome such as those present in KSS patients have been established as the primary deficiency in many of the disorders of the respiratory chain (Guenthard et al., 1995). As noted above, the 'hot spot' mtDNA deletion mutation present in skeletal muscle is also found in myocardiocytes (Anan et al., 1995; Young et al., 2005) and is present in about a third of the patients with KSS; this deletion is considered to be the cause of cardiac conduction defects (Di Nora et al., 2021). At the cellular level, mitochondrial proliferation and loss of myofibrils in heart muscle cells is connected to the cardiac pathological features (Young et al., 2005). The accumulation of mitochondria results in swelling of cardiomyocytes contributing to hypertrophy of the myocardium. A common way to detect hypertrophic, dilated, or restrictive cardiomyopathy is through echocardiography (Guenthard et al., 1995). The clinical sequence of cardiomyopathy is typically dependent on the severity of impairment of systolic and diastolic cardiac function. The reduction in systolic function and impairment of diastolic filling is caused by the hypertrophied myocardium and left ventricle which results from the proliferation of mitochondria. Therefore, decreased systolic function is the result of decreased numbers of myofibrils and 
insufficient mitochondrial function (Guenthard et al., 1995). Any patients that experience a sudden death (about 20\%) all result from cardiac events; diminished systolic function has been highly correlated with deaths before age 3 because cardiomyopathy associated with respiratory chain defects is severe and deteriorates quickly (Guenthard et al., 1995; Young et al., 2005). In a study done by Anan and colleages (1995), all of the patients that had MD the 3 with KSS all exhibited cardiac conduction disruptions such as complete AV block caused by complete right bundle branch block, infra-His block, and/or left anterior hemiblock with prolongation of the HV interval.

\section{Brain Abnormalities}

The choroid plexus (CP) is located in the ventricular system between both lateral ventricles and the third and fourth ventricles of the brain. Aside from the primary function of the CP to produce the cerebrospinal fluid (CSF) located within the ventricles, it also functions in the active transport of micronutrients (Spector \& Johanson, 2010). KSS affects the anatomical structure of the CP and its physiological function; these defects are a result of insufficient ATP concentrations to drive cellular processes such as the active transport of folate in the CP to the central nervous system. The aggregation of mutated mtDNA genomes in the CP significantly contributes to an increase of CSF protein levels. KSS is an archetype of CP failure in which the transport of folate from the blood into the CSF is inadequate and causes profound cerebral folate deficiency (Quijada-Fraile et al., 2014). In KSS, the CP epithelial cells are enlarged and granular due to eosinophilic-like granules, which are formed due to the functional consequence of not transporting adequate amounts of the essential micronutrient methyltetrahydrofolic acid (MeTHF) into the CSF (Reviewed in Spector \& Johanson, 2010). MeTHF is transported by the CSF, as well as other exogenous chemicals, to the mammalian brain; however, deficiency of MeTHF induces conspicuous anatomical changes to human CP epithelial cells. Abnormalities in brain tissue detected by magnetic resonance imaging (MRI) have been demonstrated to rise in tandem with the neurologic progression of KSS. Neuroimaging using MRI revealed lesions such as abnormalities in the brain stem, subcortical cerebral white matter, basal ganglia, and thalamus (Quijada-Fraile et al., 2014). The normal range of MeTHF CSF concentrations in plasma is 50-134 nM, with KSS patients presenting with a very low concentration of $8 \mathrm{nM}$. Therefore, KSS is considered a driver of diffuse CP failure (Spector \& Johanson, 2010).

\section{Muscle Involvement}

Many times, the common diagnoses for KSS involve clinical features such as: CSF protein levels above the normal 1 $\mathrm{g} / \mathrm{L}$, cerebellar syndrome, and skeletal muscle biopsy specimens revealing the presence of ragged-red fibers (Anan $e t$ $a l ., 1995)$. When muscle biopsy tissue of KSS patients is analyzed with Gomori's trichrome specimen stain, abnormal cells show a network of red fibers known as ragged-red fibers. The appearance of these red-ragged fibers is due to the accumulation of atypical mitochondria directly adjacent to the plasma membrane of the muscle fiber; these aggregated mitochondria induce an irregular contour to the muscle fiber producing a "ragged" appearance (Chi, 2015). Light microscopy of muscle biopsy is an ancillary diagnostic method to observe muscle-type fiber predominance and distribution as well as myofibrillar integrity (Chi, 2015). Khambatta and coworkers (2014) have determined that raggedred fibers, caused by mislocalization of morphologically aberrant mitochondria, as mentioned above, are indicative of combinatorial defects in respiratory complexes I and IV. These data provide a direct link between physiological and morphological markers of KSS pathogenesis.

\section{Treatment}

Currently, no restorative treatments are available for mtDNA disorders. As of now the treatment options available aim to maintain the overall patient health but do not restore, remove, or recompense for the detrimental mtDNA mutations (Patanan et al., 2017). The biggest challenge to restorative medicine for KSS patients is that in contrast to nuclear 
DNA (nDNA) it is very difficult to alter mtDNA sequences in mammalian cells. The only potential way that the mtDNA sequences could be repaired in such a way is through mitochondrial replacement procedures which targets future offspring. Thus, genetic counseling for these patients is necessary and involves analyzing the family history of mitochondrial disease. This helps understand what type of mutation is present as well as develop the most appropriate and effective strategy to manage the symptoms through a full mtDNA sequencing that identifies the haplogroups and variants (A. Saldaña- Martinez et al., 2019). Methods such as a mitochondrial replacement procedure, that can improve quality of life, involve the transfer of nDNA from an intact nucleus, meiotic spindle-chromosomal complex, pronucleus, or a polar body. The nDNA is removed from an ovum containing mutant maternal mtDNA into an enucleated donor ovum with wild-type mtDNA (Patanan et al., 2017).

Due to the pleiotropic nature of this disorder, it is imperative for patients that present with ptosis or chronic progressive ophthalmoplegia to have the appropriate referrals to cardiologists, endocrinologists, and ophthalmologists since various organs are involved. Considering there is a high correlation of patients with renal disease that also have mtDNA deletions, novel therapies such as the implementation of highly sensitive urinary markers to detect for tubular damage allow for an earlier intervention (Broomfield et al., 2015). There also exists the possibility that transmission of pathogenic mtDNA could be prevented by the development and implementation of alternative in vitro fertilization techniques thereby reducing the possibility of disease and alleviating patient and societal disease burden (Gorman $e t$ al., 2015).

\section{Arginine Therapy}

Arginine is known to ameliorate KSS symptoms because stroke-like episodes are known to occur due to abnormal nitric oxide flux and arginine acts as a nitric oxide donor. Furthermore, since the biosynthesis of citrulline, arginine's precursor, is an ATP-dependent process the levels of plasma arginine are found to be reduced in mitochondrial diseases (Ganetzky \& Falk, 2018). Since 2005, arginine has been used as an oral prophylactic and acute intravenous (IV) therapy to ameliorate the high mortality rate of stroke-like episodes in MD patients (Ganetzky and Falk, 2008). Furthermore, Ganetzky and Falk (2008) conducted an 8-year retrospective analysis to evaluate IV arginine use at the time of stroke-like episodes in a cohort of 71 subjects under the age of 18 presenting with MD. Out of those patients, only 9 were given a standardized regimen of $500 \mathrm{mg} / \mathrm{kg}$ IV arginine for one or more stroke-like episodes. The data demonstrated that $47 \%$ of acute stroke-like episodes showed a positive clinical response to IV arginine; whereas, the remaining $6 \%$ of episodes that showed clinical improvement could not be directly attributed solely to IV arginine therapy. Of note, however, no patient exhibited sustained clinical improvement beyond the 48 hours of treatment. Despite the limitations to the study of having a small sample size of individuals with acute stroke-like episodes who received IV arginine therapy it is still considered the largest clinical retrospective analysis (Ganetzky \& Falk, 2018).

\section{Folinic Acid Therapy}

The effects of folinic acid therapy have been explored considering that the organ most affected by folate deficiency is the brain (Quijada-Fraile et al., 2014). Due to the key role of this vitamin in brain function there is strong support for folinic acid supplementation for KSS patients. Folate is an essential methylating compound needed for proper brain metabolism. In the investigation done by Quijada-Fraile and colleagues (2014), of the eight patients studied, two cases showed neurological improvement following folinic acid therapy. While comparison of brain morphology as assessed by MRI revealed that one case had improvement in the white matter abnormalities, the rest still exhibited progression in the abnormalities of the cerebellum, subcortical cerebral white matter, and cerebral atrophy. Although it is encouraging that two cases had neurological improvements the sample size is very small, making it a limitation to the efficacy of the therapy. Of course this is also because it is an orphan disease that already has a small number of willing people to be included in studies. Thus, while early intervention with high-dose folinic acid treatment appears advisable for KSS syndrome patients to improve neurological outcomes, its efficacy is not considered affirmative. 


\section{Prospectus}

Considering that this is an orphan disease the cohort of patients available for treatment studies is very low. Hence why mouse models have been created and used for mitochondrial diseases in order to better understand the pathophysiological mechanisms that undergird the complex assortment of symptoms. Through the different mouse models representing the genetic and physiological variations in mitochondrial diseases there could be future advances into the role of mitochondrial defects (Wallace, 1999). Two decades later, modern research supports the importance of implementing full sequencing of mitochondrial genomes of patients with pleiotropic disorders such as KSS. This expansion would allow the discovery of new variants and their functional impact as part of the complex protein suppliers of ATP by the phosphorylation system (A. Saldaña- Martinez et al., 2019). By better understanding the mitochondrial genetic background among KSS patients we can adapt their treatment approach accordingly and enhance the quality of life.

Mouse models should be developed to understand why the "hot spot" deletion among KSS patients presents such different manifestations and onset of symptoms. It is crucial to develop a study that narrows down the variability between clinical phenotypes. The set of mice would have the same mtDNA common deletion and therefore provide insight as to how that deletion affects the physiology and homeostasis of the mouse. After seeing the phenotypes the mice present with the method of treatment could be tailored specifically to ameliorate that symptom. For example, by knowing that a patient has the variant nm.16519T we can ensure that part of the medications administered include mitochondrial supplements such as co-enzyme Q10, riboflavin, and L-carnitine since that variant has been directly associated with cyclic vomiting syndrome. Furthermore, research currently points towards sequencing and determining both the haplogroup and haplotype to establish the importance of pathophysiology and to track the migration and evolution of populations shown to be associated with KSS. Being able to understand the genetic ancestry as well as what ethnicities are more predisposed to developing this rare and pleiotropic mitochondrial disease would provide great insight as to what causes the disease. By knowing who is more prone and at risk to have KSS we can find the underlying problem and commonality among patients hence find a potential cure or at least develop an appropriate preventive measure. One of the hardest issues with this disease is the fact that it is phenotypically heterogenous, thus by being able to understand the connection among these patients would increase the possibility of improving the chances of survival.

The diagnostic approach for KSS is always a challenge due to the "complexity of the mitochondrial machinery (41,100 proteins), its dual genetic regulation ( $\mathrm{mt}$ and nuclear DNA), and the coexistence of mutated and wild type mtDNA (heteroplasmy)" (Mayorga et al., 2016). Thus, there is a need for better cataloguing and further analyses to generalize the standardized aspects of KSS since this mitochondrial disease of heterogenous nature makes research analysis very difficult (Lee et al., 2018). Future research seems to lean in the direction of focusing more on the size and heteroplasmy of deletions in mtDNAs since different patients present differing types, loci, intervals, and grades of heteroplasmy. Once again, the mouse models would allow for large-scale analysis of common genes relevant to the pathology and identification of the disease. For example, ragged red fibers are caused by a mutation in the mitochondrial genome encoded tRNA lysine gene; inactivation of this gene in the mouse would allow the analysis of the direct effects on mouse metabolism (Wallace 1999). Likewise, mouse models can provide additional insight into mitochondrial function since mouse cancer models with tumor cells lacking mtDNA have reduced mitochondrial function and tumor growth but somehow have the ability to restore respiration and augment tumorigenicity (Patanan et al., 2017).

"Establishing methods for removing detrimental mtDNA sequences or for transferring mitochondria with specific mtDNA sequences into cells are key initial steps in targeting the cause of mtDNA diseases" (Patanan et al., 2017). Establishing a long-term method (e.g. CRISPR-Cas9 gene editin) that will permit sequence alterations directly into mtDNA can provide improved clinical opportunities for those who have a mtDNA disease such as KSS (Patanan et al., 2017). Although there have been some transfer techniques performed successfully on mice, possible limitations to reproductive applications in humans include potential off-target effects from the reagents and manipulations used in the isolation and transfer of nDNA. Mitochondrial reverse genetics is another possible approach in deciphering the functions of mitochondrial genes and their encoded proteins based on the presence/absence of nucleotide substitutions, 
deletions, and insertions (Patanan et al., 2017). However, a significant limitation to this is the fact that the DNA repair machinery that is necessary to generate nucleotide substitutions, deletions, and insertions is devoid inside the mitochondria. To better understand the functional correlation between mitochondrial genes and nuclear genes and for the development of gene replacement therapies for mtDNA diseases, a push for mitochondrial reverse genetics is required (Patanan et al., 2017).

\section{Conclusions}

This review analyzes the correlation of the spontaneous deletion of mtDNA and the many symptoms that characterize KSS. The decreased mitochondrial function resulting from the deletion, causes cardiovascular and brain complications that increase the mortality rate of these patients. Due to the pleiotropic nature of the disorder, finding the functional impact of the many variants present in KSS patients could lead to a better outlook. Currently, efficient diagnosis and disease modifying therapies are the only possible improvements for these patients.

\section{References}

Anan, R., Nakagawa, M., Miyata, M., Higuchi, I., Nakao, S., Suehara, M., . . Tanaka, H. (1995). Cardiac Involvement in Mitochondrial Diseases. Circulation, 955-961.

Broomfield, A., Sweeney, M. G., Woodward, C. E., Fratter, C., Morris, A. M., Leonard, J. V., . . Rahman, S. (2015). Paediatric single mitochondrial DNA deletion disorders: an overlapping spectrum of disease. J Inheritable Metabolic Diseases, 445-457.

Chi, C.-S. (2015). Diagnostic Approach in Infants and Children with Mitochondrial Diseases. Pediatrics and Neonatology, 7-18.

Chinnery, P. F., DiMauro, S., Shanske, S., Schon, E. A., Zeviani, M., Mariotti, C., . . Deschauer, M. (2004). Risk of developing a mitochondrial DNA deletion disorder. Neurology, 592-596.

Di Nora C, Nalli C, Vendramin I, Livi U. (2021). Spinal cord and heart involvement in Kearns-Sayre syndrome: which link?. Neuroradiology, 63(1), 7-8.

Ganetzky, R. D., \& Falk, M. J. (2018). 8-year retrospective analysis of intravenous arginine therapy for acute metabolic strokes in pediatric mitochondrial disease. Molecular Genetics Metabolism, 301-308.

Gorman, G. S., Schaefer, A. M., Ng, Y., Gomez, N., Blakely, E. L., Alston, C. L., . . McFarland, R. (2015). Prevalence of Nuclear and Mitochondrial DNA Mutations Related to Adult Mitochondrial Disease. Annals of Neurology, 753-759.

Guenthard, J., Wyler, F., Fowler, B., \& Baumgartner, R. (1995). Cardiomyopathy in respiratory chain disorders. Activities in Disease in Childhood, 223-226.

Khambatta, S., Nguyen, D. L., Beckman, T. J., \& Wittich, C. M. (2014). Kearns-Sayre syndrome: a case of 35 adults and children. International Journal of General Medicine, 325-332. 
Lee, S.-J., Na, J.-H., Han, J., \& Lee, Y. M. (2018). Ophthalmoplegia in Mitochondrial Disease. Yonsei Medical Journal, 1190-1196.

Mayorga, L., Laurito, S. R., Loos, M. A., Eiroa, H. D., Pinho, S. d., Lubienicecki, F., . . Roque, M. (2016). Mitochondrial DNA deletions detected by Muliplex Ligation-Dependent Probe Amplification. Mitochondrial DNA, 2864-2867.

Moraes, C. T., M.Sc., DiMauro, S., Zeviani, M., Lombes, A., Shanske, S., . . Nonaka, I. (1989). Mitochondrial DNA deletions in progressive external Ophthalmoplegia and Kearns-Sayre Syndrome. The New England Journal of Medicine, 1293-1299.

Nakase, H., Moraes, C. T., Rizzuto, R., Lombes, A., DiMauro, S., \& Schon, E. A. (1990). Transcription and Translation of Deleted Miotchondrial Genomes in Kearns-Sayre Syndrome: Implications for Pathogenesis. $\mathrm{Hu}$ man Genetics, 418-427.

Patananan, A. N., Wu, T.-H., Chiou, P.-Y., \& Teitell, M. A. (2016). Modifying the Mitochondrial Genome. Cell Metabolism, 785-796.

Quijada-Fraile, P., O'Callaghan, M., Martin-Hernandez, E., Montero, R., \& Garcia-Cazorla, A. (2014). Follow-up of folinic acid supplementation for patients with cerebral folate deficiency and Kearns-Sayre syndrome . Orphanet Journal of Rare Diseases, 217-226.

Saldaña- Martinez, A., Muñoz, M. d.-S., Montoya, J., Emperador, S., Cuevas-Covarrubias, S. L.-V., \& Garcia, R. (2019). Whole sequence of the mitochondrial DNA genome. Gene 688, 171-181.

Spector, R., \& Johanson, C. E. (2010). Choroid plexus failure in the Kearns-Sayre syndrome. Cerebrospinal Fluid Research, 14-17.

Wallace, D. C. (1999). Mitochondrial Diseases in Man and Mouse. Science, 1482,1488.

Wong, L.-J. C. (2001). Recognition of mitochondrial DNA deletion syndrome with non-neuromuscular multisystemic manifestation. Genetics in Medicine, 399-404.

Yamashita, S. N., Nonaka, I., \& Goto, Y.-i. (2008). Genotype and phenotype analyses in 136 patients with single large-scale mitochondrial DNA deletions . Human Genetics, 598-606.

Young, T. J., Shah, A. K., Lee, M. H., \& Hayes, D. L. (2005). Kearns-Sayre Syndrome: A Case Report and Review of Cardiovascular Complications. Pace, 454-457.

Yu, N., Zhang, Y.-f., Zhang, K., Xie, Y., Lin, X.-j., \& Di, Q. (2016). MELAS and Kearns-Sayre overlap syndrome due to the mtDNA m. A3243G mutation and large-scale mtDNA deletions. eNeurologicalSci, 15-18.

Zhang, M., Chen, J.-L., Wang, P.-C., Fu, C.-Y., \& Peng, H. (2017). Complete Mitochondrial Genome Sequence of the Human Neuroblastoma Cell Line 751-NA . Genome Announcements. 